\title{
Trimanual Anterior Vitrectomy: A Novel Technique to Manage Vitreous Loss during Phacoemulsification
}

\author{
Michael G. Taggart R. Grant Morshedi Balamurali K. Ambati \\ John A. Moran Eye Center, University of Utah, Salt Lake City, Utah, and Harvey and \\ Bernice Jones Eye Institute, University of Arkansas, Little Rock, Ark., USA
}

\section{Key Words}

Vitrectomy · Cataract surgery · Phacoemulsification · Trimanual · Posterior capsular tear

\begin{abstract}
We report 2 cases illustrating the use of a new technique to manage vitreous loss during phacoemulsification, which we have termed 'trimanual' anterior vitrectomy. In each case, after recognizing posterior capsule tear, the remaining nuclear pieces were removed with low-parameter phacoemulsification. The remaining cortical material was then removed using bimanual irrigation and aspiration handpieces while the assistant surgeon inserted the vitrectomy probe through a separate $1-\mathrm{mm}$ limbal incision. The vitrectomy probe was held below the plane of the posterior capsule tear, used to cut the vitreous and to provide a mechanical blockade to potentially descending lens material. While this technique involves the potentially awkward simultaneous use of 3 intraocular instruments, we believe that there are several advantages over standard bimanual anterior vitrectomy.

(c) 2014 S. Karger AG, Basel
\end{abstract}

\section{Introduction}

The incidence of intraoperative posterior capsule tears varies in the literature, ranging from $0.45-3.6 \%$ even among experienced surgeons [1]. Although rare, posterior capsule tears and vitreous loss can cause serious intra- and postoperative complications such as loss of nuclear fragments into the vitreous cavity, endophthalmitis, retinal detachment as well as cystoid macular edema. After the recognition of the posterior capsule tear with vitreous loss, 
Taggart et al.: Trimanual Anterior Vitrectomy: A Novel Technique to Manage Vitreous Loss during Phacoemulsification

the safe and effective removal of vitreous from the anterior chamber is imperative to optimize surgical outcomes.

Until bimanual anterior vitrectomy is described [2-4], automated anterior vitrectomy can be performed using a vitrectomy handpiece with a coaxial irrigation sleeve. The separation of the irrigation sleeve from the vitrectomy probe marks an important advantage in the safety of anterior vitrectomy. Surgeons are now able to maintain a pressurized globe without overly hydrating the vitreous and causing a further vitreous prolapse [5]. We report a novel technique of further separating vitrectomy functions by separating the aspiration line from the vitreous cutter and inserting these 2 instruments through separate limbal incisions. We have termed this technique 'trimanual' anterior vitrectomy. To our knowledge, this technique has not yet been previously reported.

\section{Surgical Technique}

Case 1

Phacoemulsification was performed via a 2.8-mm temporal clear corneal incision using a phaco chop technique of nuclear disassembly. Once the posterior capsule tear was recognized, the anterior chamber was filled with viscoelastic, and the remaining nuclear pieces were carefully removed using low-parameter phacoemulsification. Because there was vitreous present in the anterior chamber, there was a need for an anterior vitrectomy rather than proceeding directly with the cortex removal. The phacoemulsification unit (Infiniti ${ }^{\circledR}$, Alcon Laboratories, Inc., Fort Worth, Tex., USA) was switched to the anterior vitrectomy mode, and the 20-gauge vitrectomy probe and a 21-gauge bimanual irrigation and aspiration (I/A) handpiece set were connected to the phacoemulsification unit in the following manner (fig. 1):

- The irrigation line was connected to the irrigation handpiece from the bimanual I/A set.

- The aspiration line was connected to the aspiration handpiece from the bimanual I/A set, rather than to the aspiration connector on the vitrectomy probe. The aspiration outlet tubing of the vitrectomy probe was capped.

- The pneumatic actuation line from the vitrectomy probe was connected to the phacoemulsification unit.

Two additional 1-mm paracentesis incisions were created at the 11:30 and 1:30 positions (positioned to be accessible to the assistant who was positioned superiorly on this left eye surgery). The irrigation handpiece (held in the surgeon's left hand) was inserted through the initial paracentesis incision at the 5:00 position, and the aspiration handpiece (held in the surgeon's right hand) was inserted through the 1:30 paracentesis. The vitrectomy probe was inserted by the assistant through the 11:30 paracentesis and held with the cutting port facing up, with the tip positioned posterior to the posterior capsule tear (fig. 2). The phacoemulsification unit was initially in cut-I/A mode, and the vitrectomy probe was used to cut any vitreous in the anterior chamber. The unit was then changed to I/A-cut mode, and the surgeon used the bimanual I/A handpieces to carefully strip the cortex from the remaining capsular bag while periodically entering foot position 3, if necessary, to cut vitreous with the vitrectomy probe. The vitrectomy probe was primarily held in its position posterior to the capsule defect and used secondarily to mechanically block any lens fragments that had the potential to descend posteriorly through the capsule defect. The vitrectomy probe was occasionally raised into the anterior chamber to cut vitreous strands engaged in the remaining cortical material. 
Taggart et al.: Trimanual Anterior Vitrectomy: A Novel Technique to Manage Vitreous Loss during Phacoemulsification

Once the cortex had been cleared from the eye and no vitreous remained in the anterior chamber, the vitrectomy probe and aspiration handpiece were removed from the eye, the anterior chamber was filled with viscoelastic material, and the irrigation handpiece was removed from the eye. A 3-piece hydrophobic acrylic intraocular lens was injected into the ciliary sulcus, and the optic was captured posteriorly through the intact continuous curvilinear capsulorhexis.

Case 2

Phacoemulsification was performed via a 2.8-mm temporal clear corneal incision using a phaco chop technique of nuclear disassembly. During phacoemulsification, a nasal posterior capsule tear occurred without any vitreous prolapse. Remaining nuclear fragments were removed in a low-flow technique with copious, viscoelastic protection in the anterior chamber. At the conclusion of phacoemulsification, vitreous prolapse was noted. Additional paracentesis incisions at the 7:00 and 2:00 positions were performed. As described in the first case, trimanual anterior vitrectomy was performed to remove the vitreous and cortex. However, in this case, a 23-gauge vitrectomy probe (Infiniti ${ }^{\circledR}$ Ultravit ${ }^{\circledR}$, Alcon Laboratories, Inc.) was used instead of a 20-gauge vitrectomy probe. A 3-piece hydrophobic acrylic intraocular lens was injected into the ciliary sulcus, and the optic was captured through the intact continuous curvilinear capsulorhexis.

\section{Outcomes}

Both patients later required a pars plana vitrectomy for retained epinuclear fragments (thought to have descended prior to the beginning of the anterior vitrectomy). The anterior segments remained quiet with no vitreous and a well-centered sulcus lens implant. Two weeks after surgery, the patient described in the second case had an uncorrected visual acuity of 20/25, and the patient described in the first case had a best-corrected visual acuity of $20 / 200$, which was due to geographic atrophy.

\section{Discussion}

The occurrence of posterior capsule tears cannot be eliminated, so it is imperative to be able to safely and effectively manage this complication and maximize the potential outcome. If the anterior hyaloid face ruptures and vitreous prolapses into the anterior chamber, an anterior vitrectomy is necessary. The performance of an automated anterior vitrectomy with a coaxial irrigation sleeve increases the likelihood of hydrating the vitreous and inducing further vitreous prolapses [5]. Some authors propose a 'dry' technique following posterior capsule rupture to minimize this complication [6], where all procedures following the posterior capsule rupture are performed under viscoelastic pressurization rather than irrigation.

Bimanual anterior vitrectomy also theoretically reduces the risk of hydrating the vitreous, as the irrigation cannula can be left more anteriorly in the anterior chamber while the vitrectomy probe is placed through the posterior capsule defect and used to cut the vitreous. In this system, the vitrectomy probe has both cutting and aspiration functions, with the order of the 2 functions dependent on whether the machine is in I/A-cut or cut-I/A mode. Since the same port is used for both cutting and aspirating, even surgeons using fine foot pedal control will occasionally aspirate vitreous when cutting is instead indicated, which places traction on the vitreous and increases the risk for retinal tears and/or detachment. Additionally, in our experience, attempting to remove remaining lens material (especially 
Taggart et al.: Trimanual Anterior Vitrectomy: A Novel Technique to Manage Vitreous Loss during Phacoemulsification

epinucleus and cortex) in a 'stripping' fashion with the vitrectomy probe can be difficult, as the sharp edges of the port can cut (and thereby release) the accumulated cortical material, even when the cutting function is not involved. This limits the attainable vacuum, which must be of a sufficient level to be able to strip the cortical material off of the capsular bag. In contrast, the smooth round port of the bimanual aspiration handpiece is more easily obstructable and less likely to cut the cortical material, making the removal of the remaining cortical material more efficient (fig. 3). Therefore, when a posterior capsule rupture is identified prior to the complete removal of all epinuclear and cortical material, a potentially valuable alternative may be the trimanual anterior vitrectomy technique. The remaining nuclear material, depending on the density, would likely have to be dealt with first, either with a continued low-parameter phacoemulsification or a conversion to extracapsular cataract extraction.

Another potential benefit to trimanual anterior vitrectomy is the reduced need to constantly switch between cut-I/A and I/A-cut modes. When in I/A-cut mode during bimanual anterior vitrectomy, if the surgeon encounters a significant amount of uncut vitreous, it is usually necessary to switch back to cut-I/A mode to avoid aspirating uncut vitreous. However, with trimanual anterior vitrectomy, since the cutting instrument remains below the plane of the posterior capsule defect and the aspiration tip is above, once the initial vitreous cutting/aspirating has been performed, it should rarely be necessary to switch back to cut-I/A mode since the aspirating instrument is kept well away from the vitreous. The vitrectomy probe remains posterior to the capsule defect, and the surgeon only enters foot position 3 when necessary.

In trimanual anterior vitrectomy, the performance of the aspiration and cutting functions are performed by 2 separate instruments (fig. 4). Similar to the advantages realized when irrigation was separated from automated vitrectomy handpieces, we believe that there are several advantages inherent to separating these 2 functions. However, there are some noteworthy limitations. One disadvantage of our technique is the use of a surgical assistant to hold 1 of the intraocular instruments (in each of these cases, an experienced senior ophthalmology resident with more than 150 phacoemulsification surgeries is present as the primary surgeon). In future cases requiring trimanual anterior vitrectomy, we believe it would be valuable to use an anterior chamber maintainer for the irrigation function, thus enabling the surgeon to hold the aspiration handpiece in one hand and the vitrectomy probe in the other hand. This would avoid the simultaneous manipulation of 3 instruments in the eye. If an anterior chamber maintainer is unavailable and an assistant is required to hold an instrument, it would be best to have the assistant hold the stationary irrigation handpiece. Another minor drawback is the need to create a third paracentesis incision, in addition to the second incision necessary to perform bimanual I/A. Finally, depending on the location of the incisions, posterior capsule tear and remaining lens material, the simultaneous use of 3 instruments in the anterior segment could be awkward. Although both of the patients described in this report later required 3-port pars plana vitrectomy to remove epinuclear material from the vitreous cavity, this was unrelated to the use of the trimanual anterior vitrectomy technique over the bimanual technique, as the lens material descended prior to the beginning of the anterior vitrectomy. While the technique of performing a trimanual anterior vitrectomy does not eliminate the need for a pars plana vitrectomy, it does however enable a more complete cleanup of the anterior segment.

A potential modification to our described technique would be to insert the vitrectomy probe through a pars plana incision [7], while the aspiration handpiece is inserted through a paracentesis (as described above). This modification is best left to surgeons who have experience with pars plana incisions. Another modification, as in our second case, is the use 
Taggart et al.: Trimanual Anterior Vitrectomy: A Novel Technique to Manage Vitreous Loss during Phacoemulsification

of the 23-gauge vitrectomy probe, which, in addition to the smaller diameter, supports higher cut rates [8] and may be more appropriate than the 20-gauge probe for surgeons utilizing the pars plana approach.

We believe that trimanual anterior vitrectomy is a noteworthy modification of anterior vitrectomy, a technique required to manage the rare but unavoidable complication of posterior capsule tear with vitreous prolapse. The use of the trimanual technique in our cases allowed for a more efficient removal of the remaining lens material in the anterior segment while simultaneously safely cutting and removing the prolapsed vitreous. In such cases, it is our hope that this technique allows the surgeon to maximize the chances of a successful postoperative outcome.

\section{Acknowledgements}

This work was supported in part by an unrestricted grant from Research to Prevent Blindness, Inc., New York, N.Y., USA, and by the Department of Ophthalmology and Visual Sciences, University of Utah, Salt Lake City, Utah, USA.

\section{References}

1 Wu MC, Bhandari A: Managing the broken capsule. Curr Opin Ophthalmol 2008;19:36-40.

$\rightarrow 2$ Wang HS: Management of a posterior capsule rupture in planned extracapsular cataract extraction and posterior chamber lens implantation. J Cataract Refract Surg 1986;12:73-76.

-3 Osher RH, Cionni RJ: The torn posterior capsule: its intraoperative behavior, surgical management, and long-term consequences. J Cataract Refract Surg 1990;16:490-494.

4 Kusaka S, Tsujioka M, Mano T, Tsuboi S, Ohashi Y: Two-port vitrectomy for vitreous loss during sutureless cataract surgery. Am J Ophthalmol 1994;117:533-534.

5 Koch PS: Complications of cataract and intraocular lens surgery. Curr Opin Ophthalmol 1991;2:53-60.

6 Akura J, Hatta S, Kaneda S, Ishihara M, Matsuura K, Tamai A: Management of posterior capsule rupture during phacoemulsification using the dry technique. J Cataract Refract Surg 2001;27:982-989.

-7 Chalam KV, Gupta SK, Vinjamaram S, Shah VA: Small-gauge, sutureless pars plana vitrectomy to manage vitreous loss during phacoemulsification. J Cataract Refract Surg 2003;29:1482-1486.

8 Alcon Infinti Vision System Operator's Manual. http://www.infinitivision.com/product-labeling.asp (accessed June 1, 2012). 


\section{Case Reports in \\ Ophthalmology}

\begin{tabular}{l|l}
\hline Case Rep Ophthalmol 2014;5:373-379 \\
\hline DOI: 10.1159/000369272 & $\begin{array}{l}\text { ○ 2014 S. Karger AG, Basel } \\
\text { www.karger.com/cop }\end{array}$ \\
\hline
\end{tabular}

Taggart et al.: Trimanual Anterior Vitrectomy: A Novel Technique to Manage Vitreous Loss during Phacoemulsification

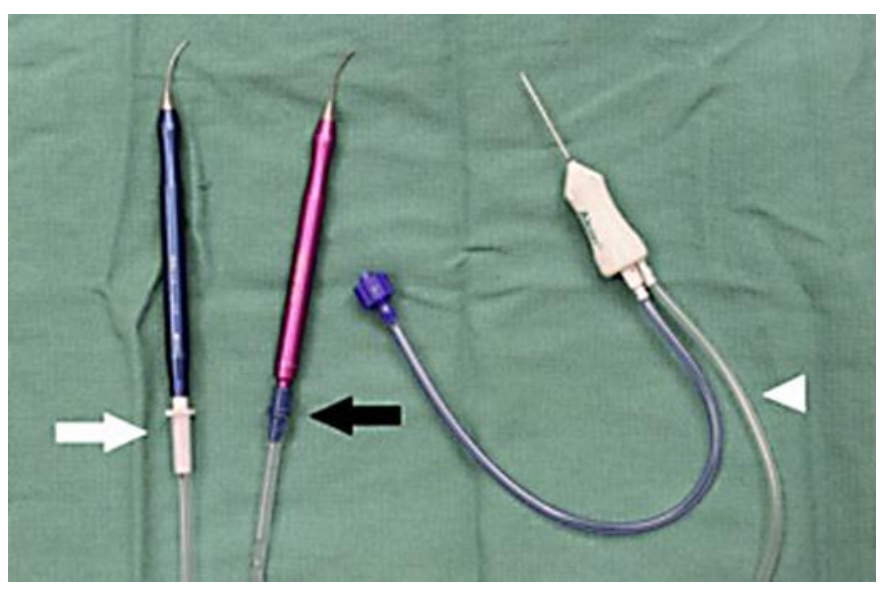

Fig. 1. Photograph showing the instrument connections. The irrigation handpiece is connected to the irrigation line (white arrow). The aspiration handpiece is connected to the aspiration line (black arrow). The pneumatic actuation line (white arrowhead) is connected to the phacoemulsification unit.

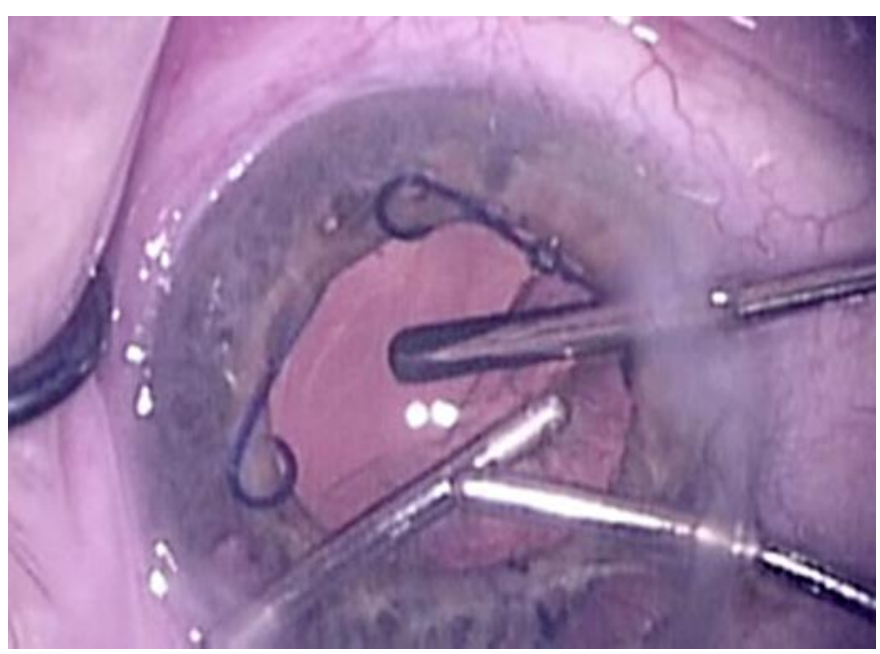

Fig. 2. Intraoperative photograph from the operating microscope (surgeon's view). The surgeon's left hand is aspirating cortex with the aspiration handpiece, and the right hand is holding the irrigation handpiece in the anterior chamber. Superiorly (right side of the photograph), the assistant is holding the vitrectomy probe with the tip posterior to the capsule defect. 
Taggart et al.: Trimanual Anterior Vitrectomy: A Novel Technique to Manage Vitreous Loss during Phacoemulsification

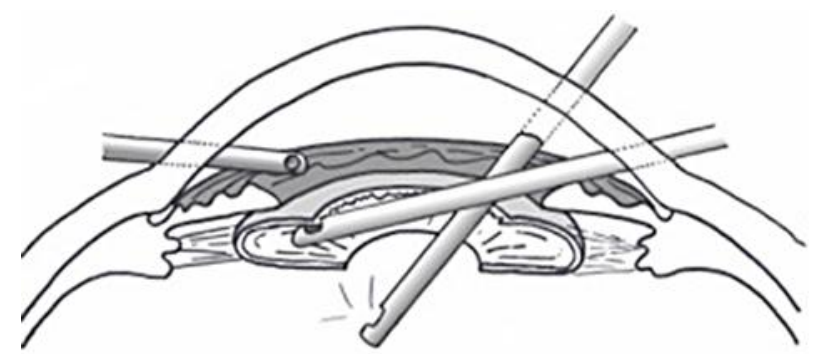

Fig. 3. Diagram showing the appropriate relative positions of the 3 instruments. The irrigation handpiece (far left) stays within the anterior chamber. The aspiration handpiece (far right) is used to remove cortex. The vitrectomy probe is held posterior to the capsule defect.

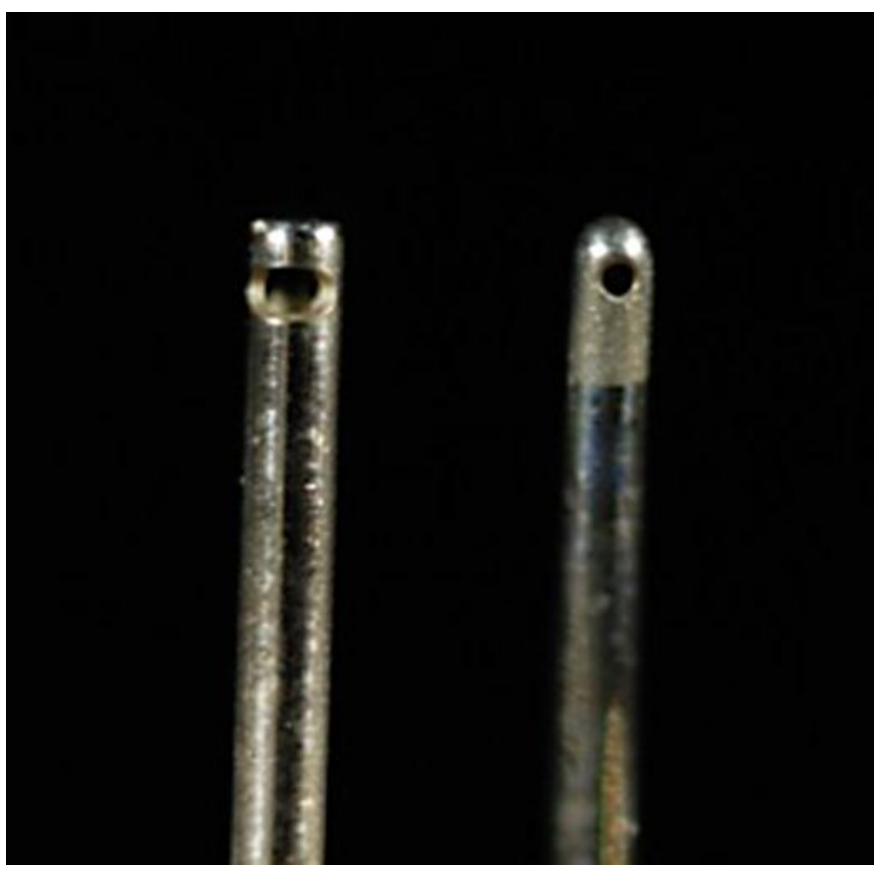

Fig. 4. Gross photograph comparing the size and shape of the aspiration ports of the 20-gauge vitrectomy probe (Alcon Laboratories, Inc.) and the 21-gauge aspiration handpiece (Katena Products, Inc., Denville, N.J., USA). 